\title{
Leiomiosarcoma uterino: valoración radiológica
}

\section{Uterine leiomyosarcoma: radiologic assessment}

\author{
Carolina Andrea Pérez Cruz, ${ }^{*}$ Carolina González Vergara, * Lizbett Hidalgo Pérez, \\ Victor Marroquín Torres, $\$$ Ludwig Isaac Arruel Caraveo $\$$
}

Citar como: Pérez CCA, González VC, Hidalgo PL, Marroquín TV, Arruel CLI. Leiomiosarcoma uterino: valoración radiológica. Acta Med Grupo Angeles. 2021; 19 (3): 376-381. https://dx.doi.org/10.35366/101731

\section{Resumen}

Introducción: El leiomiosarcoma uterino es una neoplasia maligna del músculo liso de la pared del útero, de baja supervivencia y alta mortalidad. La presencia de un sangrado uterino anormal en mujer postmenopáusica y crecimiento anormal de masa uterina son datos de alta sospecha. El diagnóstico es un desafío al ser fácilmente confundible con el leiomioma benigno y adenomiosis. Los estudios de imagen ofrecen la información adecuada para diagnóstico, planificación quirúrgica y pronóstico. Presentación del caso: Mujer de 38 años, gesta 2, partos 1, cesárea 1. Inicia su padecimiento dos meses con ocho días después de una cesárea, con presencia de masa pélvica palpable, indolora y sangrado transvaginal anormal. En exploración física, se documenta tumoración palpable no dolorosa en región pélvica de $15 \mathrm{~cm}$, sólida, irregular, móvil, con peloteo lateral y anterior hacia la cicatriz umbilical. Estudios de imagen: ultrasonido pélvico, resonancia magnética, tomografía abdominal computada trifásica y PET-CT de cuerpo entero con 18-FDG. Diagnóstico: leiomiosarcoma uterino. Tratamiento quirúrgico. Conclusión: El abordaje diagnóstico debe ser sistemático dado que es un tumor poco frecuente y agresivo y no en vano puede ser confundido con una patología benigna. Esto permite una mejor planificación terapéutica preoperatoria.

Palabras clave: Leiomiosarcoma uterino, diagnóstico radiológico, tumor uterino.

\footnotetext{
* Departamento de Radiología e Imagen.

* Departamento de Anatomía patológica.

$\S$ Cirujano Oncólogo.
}

Hospital Ángeles Mocel. México.

Correspondencia:

Dra. Carolina Andrea Pérez Cruz

Correo electrónico: caroolina_0793@hotmail.com

Aceptado: 07-09-2020.

www.medigraphic.com/actamedica

\section{Abstract}

Introduction: Uterine leiomyosarcoma is a malignant neoplasm of the smooth muscle of the uterine wall, with low survival and high mortality. Abnormal uterine bleeding in postmenopausic women and abnormal growth of uterine mass are highly suspected data. Diagnosis is challenging as it is easily confused with benign leiomyoma and adenomyosis. Imaging studies offer useful information for diagnosis, surgical planning and prognosis. Case presentation: 38 year old female, G2, P1, C1. Cesarean section three months prior to diagnosis. She began her current condition two months and eight days after her cesarean section, referring a painful palpable pelvic mass and abnormal transvaginal bleeding. Physical examination: her vital signs are stable. A palpable tumor in pelvic region of $15 \mathrm{~cm}$, solid, irregular, mobile with lateral and anterior racking towards the umbilical scar, without pain on palpation was documented. Imaging studies: pelvic ultrasound, MRI, triphasic abdominal tomography and whole body PET-CT with 18-FDG. Diagnosis: uterine leiomyosarcoma. Surgical treatment. Conclusion: The diagnostic approach must be systematic since it is a rare and aggressive tumor and not in vain can it be confused with a benign pathology. This allows for better preoperative therapeutic planning.

Keywords: Uterine leiomyosarcoma, radiological diagnosis, uterine tumor.

\section{INTRODUCCIÓN}

El leiomiosarcoma uterino es una neoplasia maligna del músculo liso de la pared del útero. Representa un $1 \%$ de todas las neoplasias uterinas y $70 \%$ de todos los sarcomas uterinos, ${ }^{1}$ ocupando el segundo lugar en frecuencia de todos los sarcomas. ${ }^{2}$ La incidencia aumenta en edades mayores de 40 años, con otros factores de riesgo como la raza negra, obesidad, diabetes tipo II, uso de tamoxifeno por más de cinco años y mutación del gen $\mathrm{p} 53 .^{1}$ 
El leiomiosarcoma presenta un comportamiento agresivo por su diseminación linfovascular temprana a estructuras pélvicas contiguas y diseminación pulmonar a distancia, condicionando alta mortalidad y recidiva, con una tasa de supervivencia de cinco años en promedio en todas las etapas de la enfermedad. ${ }^{2}$

El diagnóstico preoperatorio es un desafío, ya que el leiomiosarcoma es fácilmente confundible con el leiomioma benigno y adenomiosis, ${ }^{2}$ las cuales son patologías de alta frecuencia en mujeres en edad reproductiva ${ }^{3} y$ comparten presentación clínica como masa uterina y sangrado transvaginal anormal. Sin embargo, hay datos que dirigen el diagnóstico a leiomiosarcoma, como la edad en postmenopausia y el crecimiento anormal de masa uterina considerado más de $6 \mathrm{~cm}$ en seis a 12 meses. ${ }^{1,2}$

Los estudios de imagen tienen un papel preponderante para caracterizar la tumoración uterina, aportando información útil para la planificación quirúrgica, o bien, realizar tratamiento conservador dependiendo de su potencial maligno. ${ }^{3}$

El ultrasonido en modo B como estudio inicial tiene como utilidad calcular el volumen aproximado de la lesión, y al agregar la herramienta Doppler pulsado y color permiten valorar vascularidad para tener datos indirectos de malignidad. ${ }^{2}$ Sin embargo, en masas uterinas de gran tamaño, algunas variables como poca experiencia del operador, útero en retroversión y múltiples tumoraciones limitan la evaluación ultrasonográfica. 3,4

La tomografía axial computada es un estudio que sirve para etapificación oncológica de forma general, aunque no proporciona una adecuada resolución en lesiones uterinas y es un mal predictor para metástasis ganglionares, por lo que su uso no se considera de forma rutinaria en el diagnóstico preoperatorio de leiomiosarcoma uterino; no obstante, con las nuevas técnicas de angiotomografía y equipos helicoidales que nos ofrecen imágenes de muy alta resolución, ha tenido cambios que la convierten en una excelente herramienta de imagen. Su papel en la evaluación de enfermedad metastásica avanzada tiene baja sensibilidad por la diseminación linfovascular temprana. . $^{3,4}$

La tomografía por emisión de positrones combinada con la tomografía computada (PET/CT) proporciona datos anatómicos y valoración del metabolismo de la glucosa de las células malignas uterinas, representándose como alta captación de 18-fluorodesoxiglucosa (18-FDG) en el sitio. Su utilidad es muy alta, ya que permite identificar datos de malignidad, búsqueda de metástasis o para la recurrencia temprana postratamiento, la cual en al menos la mitad de pacientes con sarcoma uterino dura en promedio dos años después del tratamiento inicial. 4,5

La resonancia magnética (RM) es el estudio de elección para el diagnóstico preoperatorio, ya que permite la diferenciación de lesiones benignas y malignas de forma general; sin embargo, presenta algunas limitaciones, ya que no existen criterios patognomónicos. ${ }^{4}$ El leiomiosarcoma se manifiesta como una masa miometrial de gran tamaño, heterogénea, con bordes definidos nodulares y en algunos casos con infiltración a otros tejidos, con hemorragia y necrosis quística interna, de hipointensidad heterogénea en imagen ponderada en T1 y señal visible en más de $50 \%$ del tumor con intensidad intermedia a alta en T2 debido a la alta vascularidad y degeneración celular, a diferencia del leiomioma, que se presenta como masa redondeada, focal y circunscrita, que se observa isointensa en $\mathrm{T} 1$ e hipointensa en $\mathrm{T} 2$ comparada con la intensidad del miometrio. ${ }^{2,4}$ Hay leiomiomas que presentan alta vascularidad y degeneración mixoide o quística que pueden observarse hiperintensos en T2, los cuales son el principal diagnóstico diferencial por la similitud en imagen de RM con el leiomiosarcoma, por lo que el contraste endovenoso Gd-DTPA (gadolinio ácido dietilenotriamina penta-acético) debe ser parte de la rutina diagnóstica para distinguir la degeneración celular, con mínimo realce. ${ }^{3,4}$ La secuencia de difusión debe emplearse porque permite la delineación de lesiones malignas con restricción en la difusión visible como hiperintensidades a diferencia de los leiomiomas, que no restringen a la difusión y se observan hipointensos. ${ }^{4}$ Esta secuencia permite la medición de los valores de coeficiente de difusión aparente (ADC). 2,4

La importancia del diagnóstico incide en la toma de decisiones para el evento quirúrgico. En pacientes en etapa temprana, el tratamiento de elección que ayuda a aumentar la supervivencia es la resección quirúrgica completa del tumor con márgenes negativos como terapia primaria. ${ }^{1}$

\section{PRESENTACIÓN DEL CASO}

Se presenta el caso de una mujer de 38 años, originaria y residente de la Ciudad de México, con escolaridad profesional, sin antecedentes heredofamiliares relacionados con neoplasias.

Antecedentes de importancia: fumadora de los 18 a los 33 años, con índice tabáquico de 11.

Menarca a los 12 años con ritmo $28 \times 5$, ha utilizado como método anticonceptivo un dispositivo intrauterino (Mirena ${ }^{\circledR}$ ) durante los últimos tres años, citología vaginal un año previo dentro de límites normales. G2, P1, C1. La cesárea tres meses previos al diagnóstico, realizada fuera de nuestra institución, por lo que no se cuenta con el testimonio médico de las características uterinas de la cesárea.

Durante la última gesta, se realizaron ultrasonidos de control obstétrico a las 13.2 y 23.1 semanas, en ambos 
se documentó una lesión ocupativa miometrial de $5 \mathrm{~cm}$ de diámetro, catalogada como mioma intramural, al cual no se le dio manejo médico por reportarse como lesión benigna.

Inicia su padecimiento actual dos meses con ocho días después de su cesárea, por presencia de masa pélvica palpable, indolora y sangrado transvaginal anormal. A la exploración física con signos vitales estables. Se documenta tumoración palpable en región pélvica de $15 \mathrm{~cm}$ aproximadamente, sólida, irregular, móvil con peloteo lateral y anterior hacia la cicatriz umbilical, sin dolor a la palpación.

Se realiza ultrasonido pélvico que reporta tumoración sólida uterina de ecogenicidad heterogénea que mide 13.4 $\times 13.1 \times 12.5 \mathrm{~cm}$, con áreas de degeneración quística, sugiriendo miomatosis intramural de grandes elementos (Figura 1).

Se realiza resonancia magnética de pelvis con secuencias T1, T2, difusión y T1 contrastada que demuestran neoplasia uterina, que abarca desde el cuello hasta el fondo, de bordes lobulados y márgenes mal definidos, con eje mayor de $13.3 \mathrm{~cm}$ y un volumen aproximado de $919 \mathrm{~cm}^{3}$, con intensidad de señal baja en T1 e intensidad de señal heterogénea intermedia-alta en T2 con extensas zonas de necrosis central, áreas sólidas hipervasculares y zonas de hemorragia que sugieren el diagnóstico de leiomiosarcoma uterino (Figura 2).

Se observó trombosis parcial y extensión vascular tumoral de vena iliaca interna derecha, flujo lento en vena iliaca externa y femoral derecha.

Se realiza angiotomografía toracoabdominal complementaria para valorar extensión neoplásica, que además de corroborar los hallazgos de resonancia magnética, evidencia finas calcificaciones tumorales, tinción tumoral arterial miometrial con shunts arteriovenosos que condicionan drenaje venoso anómalo temprano hacia la vena ovárica izquierda, la cual persiste dilatada con un calibre máximo de $13 \mathrm{~mm}$ hasta su desembocadura en la vena renal izquierda. Trombosis tumoral de la vena iliaca interna derecha con un calibre máximo de $26 \mathrm{~mm}$, que se extiende hacia la desembocadura de la vena cava inferior (Figura 3). Uréteres dilatados con desplazamiento lateral, sin datos de hidronefrosis asociada.

Nódulo pulmonar del segmento basal posterior del pulmón derecho adherido a la pleura, sin engrosamiento de ésta, con un diámetro de $9 \mathrm{~mm}$, sin proyecciones estelares con valores de atenuación subsólidos.

Por el hallazgo del nódulo pulmonar, se decide realizar como complemento PET-CT de cuerpo entero con 18-FDG que corrobora el tumor dependiente del útero, de densidad heterogénea, con zonas hipodensas e hipometabólicas, con relación a necrosis. Se asocia a incremento anormal del metabolismo de forma irregular y heterogénea con SUV máximo de 5.26 que en fase tardía disminuye su metabolismo hasta SUV máximo de 4.98. El comportamiento metabólico sugiere neoplasia de bajo grado (Figura 3). Nódulo pulmonar se reporta sin metabolismo.

Exámenes de laboratorio: hemoglobina $14.5 \mathrm{~g} / \mathrm{dL}$, hematocrito $46.5 \%$, plaquetas $420 \times 10^{3} / \mu \mathrm{L}$, leucocitos 10.7 $\times 10^{3} / \mu \mathrm{L}$, neutrófilos $74.7 \%$, linfocitos $18.8 \%$, glucosa 116 $\mathrm{mg} / \mathrm{dL}$, urea $28.7 \mathrm{mg} / \mathrm{dL}$, creatinina $0.74 \mathrm{mg} / \mathrm{dL}$, colesterol $216 \mathrm{mg} / \mathrm{dL}$. Marcadores tumorales: alfafetoproteína 3.4 $\mathrm{ng} / \mathrm{mL}$, antígeno carcinoembrionario: $1.0 \mathrm{ng} / \mathrm{mL}$, ambos dentro de parámetros normales. Con base en el cuadro
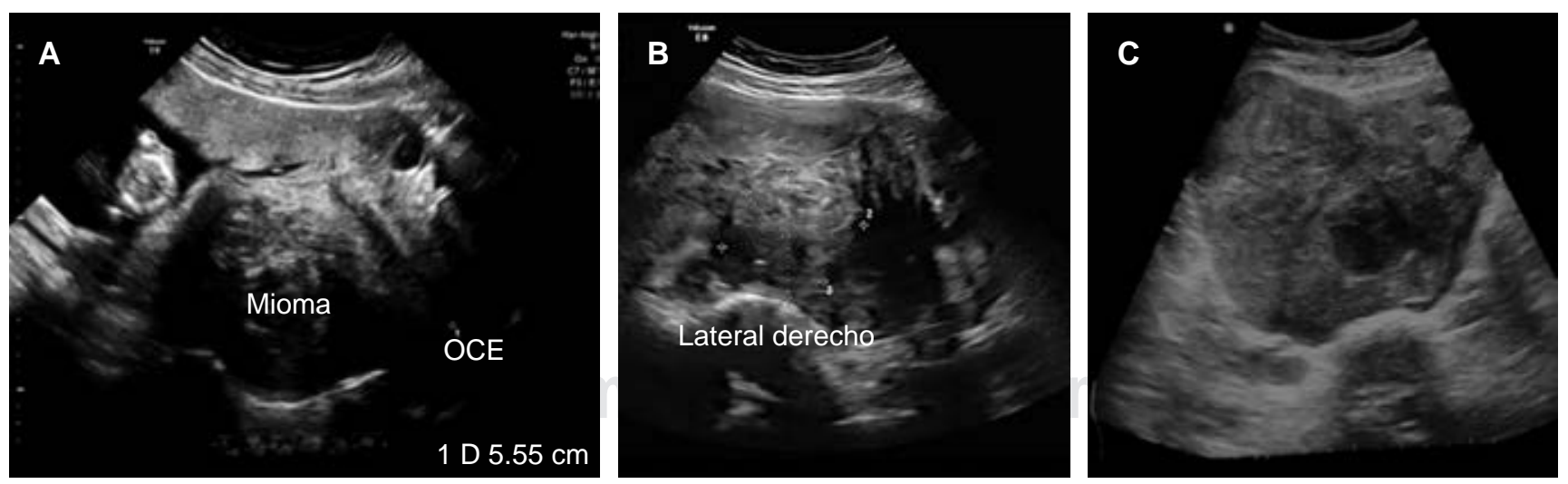

Figura 1: A) Ultrasonido obstétrico a las 13.2 semanas de gestación con lesión tumoral de 5.0 cm de diámetro interpretada en su momento como mioma uterino corporal posterior. B) Ultrasonido obstétrico a las 23.1 semanas de gestación, con lesión tumoral miometrial sin modificación al comparar con estudio anterior. C) Ultrasonido pélvico realizado a los dos meses postparto con tumoración sólida uterina de ecogenicidad heterogénea de $13.4 \times 13.1 \times 12.5$ cm en sus diámetros máximos, con áreas de degeneración quística, sus dimensiones se triplicaron. 

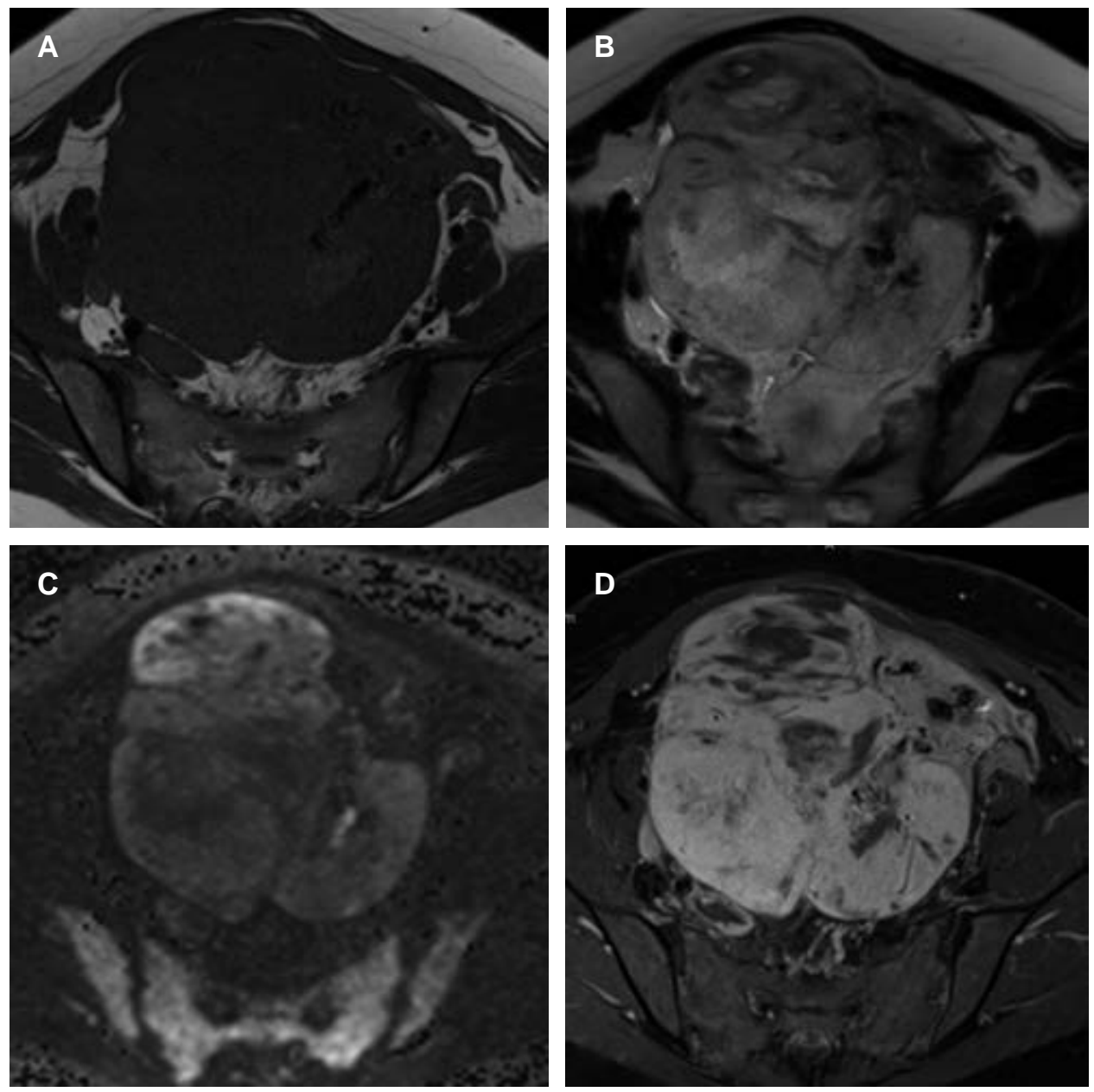

Figura 2:

A) Resonancia magnética de pelvis ponderada en $\mathrm{T} 1$, con señal de intensidad heterogénea baja en neoplasia uterina de bordes lobulados, que abarca desde el cuello hasta el fondo. B) Resonancia magnética de pelvis ponderada en T2 con señal de intensidad alta a expensas de extensas zonas de necrosis central y áreas sólidas de intensidad intermedia con distribución periférica. C) Secuencia de difusión que muestra distintos grados de restricción en la neoplasia uterina. D) Secuencia de supresión grasa en T1 con realce tumoral posterior a la aplicación de gadolinio IV. clínico y los estudios de imagen, se concluye el diagnóstico preoperatorio de leiomiosarcoma uterino.

La paciente es tratada quirúrgicamente con estudio transoperatorio que reporta una neoplasia fusocelular de alto grado compatible con sarcoma, el diagnóstico definitivo reporta leiomiosarcoma del cuerpo uterino de alto grado (15 a 17 mitosis en 10 campos de $40 \times$ ) y necrosis tumoral en $15 \%$. Trombo intramural con leiomiosarcoma y necrosis coagulativa extensa.

\section{DISCUSIÓN}

Como se ha demostrado en el caso descrito, el diagnóstico preoperatorio de leiomiosarcoma uterino se realiza con base en la sospecha clínica y los estudios de imagen, debe ser considerado como diagnóstico diferencial en toda paciente que presente un cuadro clínico caracterizado por sangrado uterino anormal y crecimiento de masa uterina en edad postmenopáusica, en esta investigación fue atípico ya que la paciente tiene 38 años. Con esta mención, queremos enfatizar la importancia del seguimiento por imagen y valoración complementaria en cualquier lesión uterina como leiomiomas benignos y adenomiosis sin importar la edad.

Como ya se ha mencionado, el leiomiosarcoma representa un $1 \%$ de todas las neoplasias uterinas y $70 \%$ de todos los sarcomas uterinos.

El abordaje diagnóstico debe ser sistemático y oportuno debido a la baja supervivencia, además de ofrecer el tratamiento adecuado, el cual requiere apoyo de los estudios de imagen para su planificación temprana. El estudio de imagen de elección es la resonancia magnética; sin embargo, debido a la baja disponibilidad en los centros hospitalarios, se puede hacer uso de otros métodos como ya se ha referido.

La angiotomografía ofrece información muy valiosa respecto a las calcificaciones tumorales y al comportamiento vascular intrínseco de la neoplasia, así como el entorno pélvico vascular arterial y venoso que determina en gran medida la planeación quirúrgica. 
Las características moleculares en el estudio de PET-CT orientaron a una neoplasia de bajo grado, pero resaltó el hecho de que no fue compatible con el hallazgo histopatológico de alto grado, aunque el radioisótopo utilizado 18-FDG es genérico. De igual manera, el nódulo pulmonar se reportó sin metabolismo, dato relevante para la decisión quirúrgica. Es un estudio que no debe evitarse, sobre todo en la evaluación de enfermedad tumoral pélvica y a distancia principalmente.
La corroboración histopatológica en las lesiones uterinas es esencial para el diagnóstico final, ya que como se demostró en el caso presentado, la confusión con lesiones benignas ocurre con alta frecuencia $y$, sin los estudios de imagen adecuados, un diagnóstico no concluyente puede retrasar el tratamiento, lo cual sería un factor de mal pronóstico.

Las características reportadas en el diagnóstico histopatológico engloban macroscópicamente una masa de gran
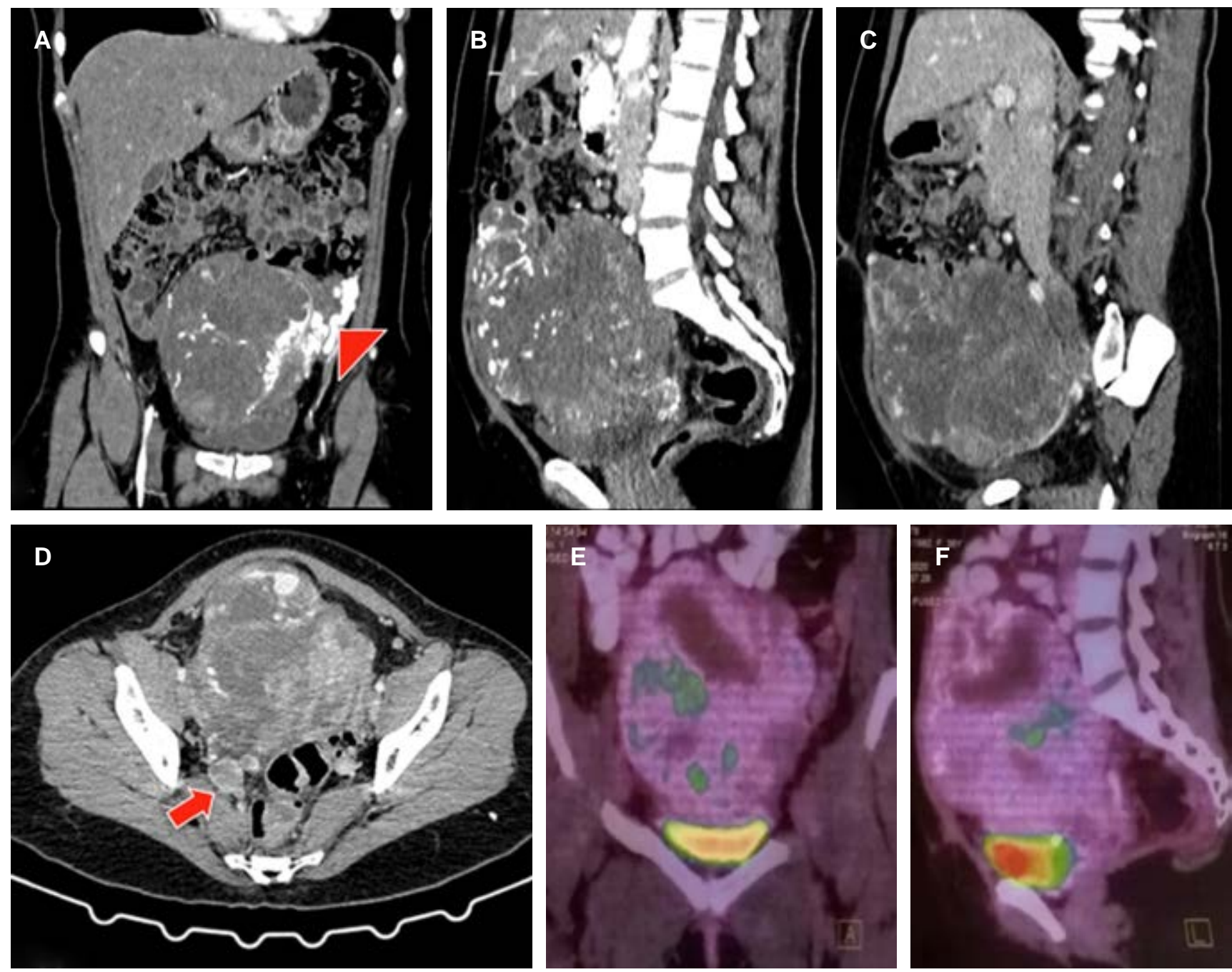

Figura 3: A y B) Corte coronal y sagital de tomografía computarizada en fase arterial con neoplasia uterina de contornos lobulados, con tinción tumoral arterial miometrial con shunts arteriovenosos que condicionan drenaje venoso anómalo temprano hacia la vena ovárica izquierda (punta de flecha). C) Corte sagital de tomografía computarizada en fase venosa con neoplasia uterina con grandes áreas de necrosis, que contacta en su borde inferior con la pared superior de la vejiga, en su borde anterior con el músculo recto abdominal y en su pared posterior con la columna lumbar. D) Corte axial de tomografía computarizada en fase venosa que demuestra trombo que involucra vena iliaca externa e interna derechas. (flecha). E) Tomografía por emisión de positrones combinada con la tomografía computada en corte coronal en donde se observa tumor dependiente del útero, de densidad heterogénea, con zonas hipometabólicas en relación a necrosis. F) Tomografía por emisión de positrones combinada con la tomografía computada en corte sagital con incremento anormal del metabolismo de forma irregular y heterogénea. 
tamaño, con zonas de hemorragia y necrosis y microscópicamente mitosis $>10$ por campo, zonas de necrosis coagulativa y atipia celular, conocidos como los criterios de Stanford para riesgo de diseminación metastásica. ${ }^{6}$ En esta investigación se reportó leiomiosarcoma del cuerpo uterino de alto grado (15 a 17 mitosis en 10 campos de $40 \times)$ y necrosis tumoral en 15\%, dos de los tres datos microscópicos que indican un riesgo metastásico de más de $10 \%$, por lo que los estudios de imagen de seguimiento no deben evitarse.

\section{CONCLUSIÓN}

Los casos con un diagnóstico precipitado o deficiente con un leiomiosarcoma insospechado, al cual se le ha confundido con leiomiomatosis benigna, tienen el riesgo de retrasar el tratamiento y/o una planificación quirúrgica incorrecta que empeora el pronóstico.

\section{REFERENCIAS}

1. Ricci S, Stone RL, Fader AN. Uterine leiomyosarcoma: epidemiology, contemporary treatment strategies and the impact of uterine morcellation. Gynecol Oncol. 2017; 145 (1): 208-216. doi: 10.1016/j. ygyno.2017.02.019.

2. $\mathrm{Wu} \mathrm{TI}$, Yen TC, Lai CH. Clinical presentation and diagnosis of uterine sarcoma, including imaging. Best Pract Res Clin Obstet Gynaecol. 2011; 25 (6): 681-689. doi: 10.1016/j.bpobgyn.2011.07.002.

3. Tanaka YO, Nishida M, Tsunoda H, Okamoto Y, Yoshikawa H. Smooth muscle tumors of uncertain malignant potential and leiomyosarcomas of the uterus: MR findings. J Magn Reson Imaging. 2004; 20 (6): 9981007. doi: 10.1002/jmri.20207.

4. DeMulder D, Ascher SM. Uterine leiomyosarcoma: can MRI differentiate leiomyosarcoma from benign leiomyoma before treatment? AJR Am J Roentgenol. 2018; 211 (6): 1405-1415. doi: 10.2214/AJR.17.19234.

5. Park JY, Kim EN, Kim DY, Suh DS, Kim JH, Kim YM et al. Role of PET or $\mathrm{PET} / \mathrm{CT}$ in the post-therapy surveillance of uterine sarcoma. Gynecol Oncol. 2008; 109 (2): 255-262. doi: 10.1016/j.ygyno.2008.01.030.

6. Silverberg SG, Kurman RJ. Tumors of the uterine corpus and gestational trophoblastic disease. Atlas of tumor pathology [electronic fascicle version 2]. Washington, DC: Armed Forces Institute of Pathology, 1992. 